\title{
Reinforcement-Driven Spread of Innovations and Fads
}

\author{
P. L. Krapivsky ${ }^{1}$ \\ ${ }^{1}$ Department of Physics, Boston University, Boston, MA, USA, 02215
}

\section{S. Redner ${ }^{2}$ and D. Volovik ${ }^{2}$}

${ }^{2}$ Center for Polymer Studies and Department of Physics, Boston University, Boston, Massachusetts 02215, USA

\begin{abstract}
We investigate how social reinforcement drives the spread of permanent innovations and transient fads. We account for social reinforcement by endowing each individual with $M+1$ possible awareness states $0,1,2, \ldots, M$, with state $M$ corresponding to adopting an innovation. An individual with awareness $k<M$ increases to $k+1$ by interacting with an adopter. Starting with a single adopter, the time for an initially unaware population that consists of $N$ individuals to adopt an innovation grows as $\ln N$ for $M=1$, and as $N^{1-1 / M}$ for $M>1$. When individuals can abandon the innovation at rate $\lambda$, the population fraction that remains clueless about the fad undergoes a phase transition at $\lambda_{c}$; this transition is second order for $M=1$ and first order for $M>1$, with macroscopic fluctuations accompanying the latter. The time for the fad to disappear has an intriguing non-monotonic dependence on $\lambda$.
\end{abstract}

PACS numbers: 02.50.-r, 05.40.-a, 89.65.-s, 89.75.Da 


\section{Introduction}

Disease propagation [1, the spread of technological innovations [2, 3, 4, 5, 6], and outbreaks of social and political unrest [7, 8] are all driven by contagion. In this work, we investigate how the mechanism of social reinforcement affects the contagiondriven evolution of permanent innovations and transient fads in a simple agent-based model. Social reinforcement means that an individual requires multiple prompts from acquaintances before adopting an innovation. This mechanism was found to foster the adoption of a desired behavior in a controlled online social network [9]. Social reinforcement stands in stark contrast to classical models of epidemics [1], where a susceptible individual can become infected by a single exposure to the infection. Despite the ubiquity of social reinforcement, this mechanism has been explored only cursorily in previous studies of contagion spread [10, 11, 12, 13, 14, 15, 16].

In our models, awareness is assumed to have a finite number of possible states. We quantify this awareness by a variable that ranges over the $M+1$ values, $0,1,2, \ldots, M$. We define an individual with awareness 0 as being susceptible, while an individual moves closer to adopting the innovation as his/her awareness value increases. Adoption of the innovation occurs when an individual reaches the highest awareness value $M$. The population evolves by repeated interactions between two random individuals. In each interaction with an adopter, someone with awareness $k<M$ advances to awareness $k+1$, while there are no state changes when two non-adopters interact. In our innovation model, an innovation is adopted permanently; in our fad model, an adopter abandons the fad at a rate $\lambda$ so that it eventually becomes passé.

\section{Permanent Innovations}

We begin with the simplest situation of no reinforcement [3, 4, 5, 6], namely, a population with two classes of individuals: susceptible (state 0) and adopters (state 1). Whenever a susceptible individual and an adopter meet, the former is converted to an adopter via $0+1 \rightarrow 1+1$. The rate equations that give the evolution of a homogeneous and well-mixed population (the mean-field limit) are:

$$
\dot{n}_{0}=-n_{0} n_{1}, \quad \dot{n}_{1}=n_{0} n_{1} .
$$

We generically assume that the evolution begins with a small fraction of adopters in an otherwise susceptible population: $n_{1}(0)=\rho \ll 1, n_{0}(0)=1-\rho$. For this initial condition the solution to the rate equations is (Fig. 1)

$$
n_{0}=\frac{(1-\rho) e^{-t}}{\rho+(1-\rho) e^{-t}}, \quad n_{1}=\frac{\rho}{\rho+(1-\rho) e^{-t}} .
$$

We define the emergence time $t_{*}$ of the innovation by the criterion that half of

the population has become adopters; $n_{0}\left(t_{*}\right)=n_{1}\left(t_{*}\right)=\frac{1}{2}$ From Eqs. (2), we have $t_{*} \simeq \ln (1 / \rho)$. Ultimately everyone is an adopter; we estimate the resulting completion 

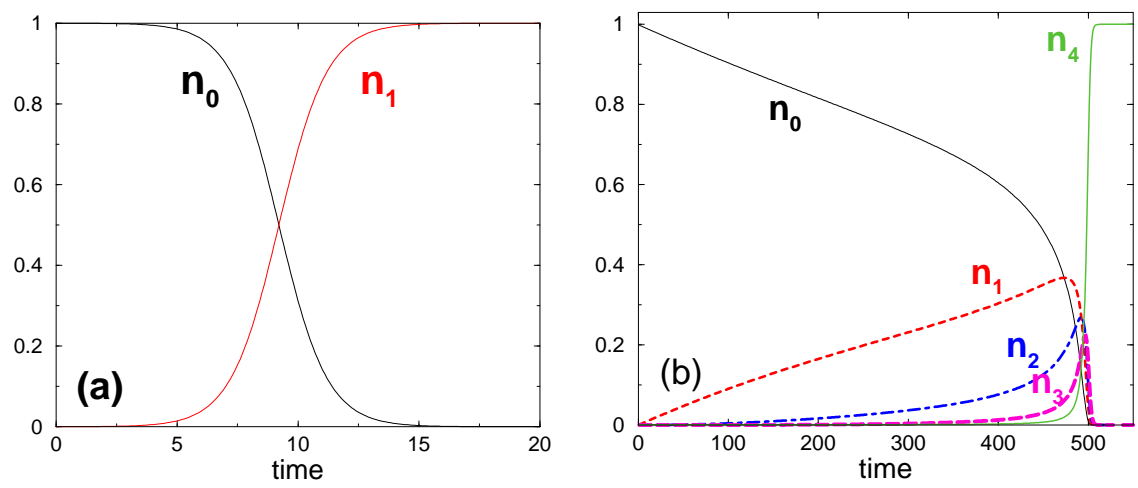

Figure 1. Time dependence of the densities $n_{k}$ by numerical integration of the rate equations for a population of size $N=10^{4}$, in which $n_{M}(0)=\rho$ and $n_{0}(0)=1-\rho$, with $\rho=\frac{1}{N}$. Shown are the cases (a) $M=1$ and (b) $M=4$.

time $T$ from $n_{1}(T)=1-\frac{1}{N}$, corresponding to all but one individual in a population of size $N$ adopting the innovation; this criterion gives $T \simeq \ln (N / \rho)$ [17].

We now implement social reinforcement by requiring individuals to move to progressively higher awareness states before adoption ultimately occurs. Possible examples of such progressions include: not owning a TV, owning a black \& white TV, owning a color TV [18], or no cell phone, dumb cell phone, smart phone, etc. We first treat the simplest example of reinforcement which is the case of $M=2$. Here there are three classes of individuals: susceptible (state 0), aware (state 1), and adopter (state 2), with respective densities $n_{0}, n_{1}$, and $n_{2}$. In an interaction with an adopter, a susceptible person becomes aware $(2+0 \rightarrow 2+1)$, while an aware person adopts the innovation $(2+1 \rightarrow 2+2)$. All other interactions do not change individual states. When the rates of all processes are the same, the rate equations are:

$$
\dot{n}_{0}=-n_{0} n_{2}, \quad \dot{n}_{1}=n_{0} n_{2}-n_{1} n_{2}, \quad \dot{n}_{2}=n_{1} n_{2} .
$$

To solve these equations we introduce the internal time $\tau=\int_{0}^{t} d t^{\prime} n_{2}\left(t^{\prime}\right)$ to simplify Eqs. (3) to a linear system, whose solution, for the generic initial condition $n_{2}(0)=\rho$, $n_{1}(0)=0, n_{0}(0)=1-\rho$, is

$$
\begin{aligned}
& n_{0}=(1-\rho) e^{-\tau}, \\
& n_{1}=(1-\rho) \tau e^{-\tau}, \\
& n_{2}=1-(1-\rho)(1+\tau) e^{-\tau} .
\end{aligned}
$$

We now define the emergence of the innovation as the point where $n_{1}$ passes through a maximum (Fig. 1(b)). This yields $\tau_{*}=1$, from which the corresponding emergence time $t_{*}$ is given by

$$
t_{*}=\int_{0}^{1} \frac{d x}{n_{2}(x)}=\int_{0}^{1} \frac{d x}{1-(1-\rho)(1+x) e^{-x}} .
$$

When $\rho \ll 1$, the asymptotic behavior of the integral is

$$
t_{*} \simeq \frac{1}{\sqrt{\rho}} \int_{0}^{1 / \sqrt{\rho}} \frac{d y}{1+y^{2} / 2} \simeq \frac{\pi}{\sqrt{2 \rho}},
$$


where $y=x / \sqrt{\rho}$, and sub-leading terms are of order of one. For a single innovator in a population of size $N$ (corresponding to initial density $\rho=\frac{1}{N}$ ), the $N$ dependence of the emergence time is

$$
t_{*}=\frac{\pi}{\sqrt{2}} N^{1 / 2}+\mathcal{O}(1) .
$$

Thus reinforcement changes the emergence time from a logarithmic to a power-law $N$ dependence (Fig. 1). Using the criterion $n_{2}(T)=1-\frac{1}{N}$, we estimate the completion time to be $T=\pi \sqrt{N / 2}+\ln N$ to lowest order [19]. Thus once the innovation emerges, it takes little additional time before it is complete.

For an arbitrary number of intermediate states, an individual with awareness $k$ increases to $k+1$ by interacting with a adopter, $[M]+[k] \rightarrow[M]+[k+1]$, with $k=0,1, \ldots, M-1$, while all other interactions do not change individual states. The corresponding rate equations are

$$
\begin{aligned}
& \dot{n}_{0}=-n_{M} n_{0}, \\
& \dot{n}_{k}=n_{M}\left(n_{k-1}-n_{k}\right), \quad k=1, \ldots, M-1, \\
& \dot{n}_{M}=n_{M} n_{M-1} .
\end{aligned}
$$

By again introducing the internal time $\tau=\int_{0}^{t} d t^{\prime} n_{M}\left(t^{\prime}\right)$, we reduce Eqs. (7) to a linear system whose solution is

$$
\begin{aligned}
& n_{j}=(1-\rho) \frac{\tau^{j}}{j !} e^{-\tau}, \quad j=0, \ldots, M-1, \\
& n_{M}=1-(1-\rho) \sum_{j=0}^{M-1} \frac{\tau^{j}}{j !} e^{-\tau} .
\end{aligned}
$$

In analogy with the case of $M=2$, the innovation emerges at $\tau=1$, where $n_{1}$ passes through a maximum (generally, each $n_{j}$ passes through a maximum at $\tau=j$ ). To obtain explicit time dependences, we must recast $\tau$ in terms of the physical time via $t=\int_{0}^{\tau} d x / n_{M}(x)$. Applying the same steps as above and setting $\rho=\frac{1}{N}$, we find the emergence time

$$
t_{*}=\frac{\pi(M !)^{1 / M}}{M \sin (\pi / M)} \times N^{1-1 / M} .
$$

Thus increasing the number of intermediate states $M$ progressively delays innovation

emergence, as the exponent $1-\frac{1}{M}$ approaches 1 as $M$ becomes large (Fig. 1(c)).

\section{Transient Fads}

Transient fads arise when adopters can independently abandon the innovation at rate $\lambda>0$. In this case, the innovation can spread to some degree before it is abandoned and fades away. The extent of the fad and its lifetime fundamentally depend on the abandonment rate. Thus the population at infinite time consists of adopters who abandoned the fad and individuals who are forever stuck in intermediate awareness states because of the absence of catalyzing adopters. Of particular interest are the 
clueless individuals who were never exposed to the fad while it was active. Their fraction, defined as $c_{\infty}(\lambda) \equiv n_{0}(t=\infty)$, characterizes the competing influences of contagion and fad abandonment [20]. For an infinite population, $c_{\infty}$ undergoes a continuous transition as a function of $\lambda$ for $M=1$, but a discontinuous transition for $M \geq 2$. Moreover, the time to reach the final state varies non-monotonically with $\lambda$.
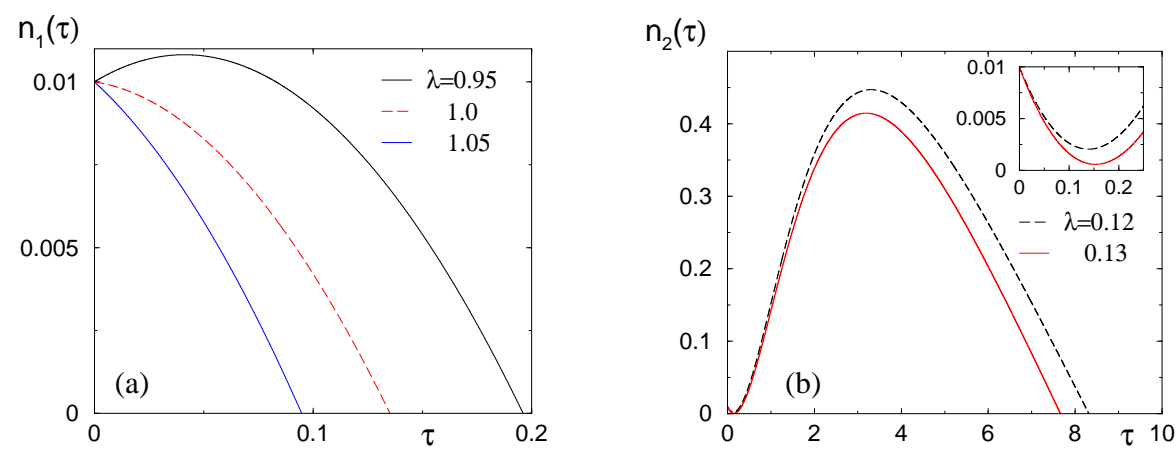

Figure 2. Dependence of $n_{M}(\tau)$ versus $\tau$ for: (a) no reinforcement $(M=1)$, and (b) reinforcement, with one intermediate state $(M=2)$, Eqs. (10) and (11) respectively. The inset in (b) shows the near tangency of $n_{2}(\tau)$ versus $\tau$ for $\lambda=0.13$.

The case of no reinforcement coincides with the classic SIR epidemic model [1] with the identifications: adopter $\leftrightarrow$ infected, abandoner $\leftrightarrow$ recovered, while the meaning of susceptible is the same in both models. The rate equations are $\dot{n}_{0}=-n_{0} n_{1}$, $\dot{n}_{1}=n_{0} n_{1}-\lambda n_{1}$, with solution

$$
n_{0}=(1-\rho) e^{-\tau}, \quad n_{1}=1-\lambda \tau-(1-\rho) e^{-\tau},
$$

where $\tau=\int_{0}^{t} d t^{\prime} n_{1}\left(t^{\prime}\right)$. The evolution ceases at an internal stopping time $\tau_{\infty}$ defined by $n_{1}\left(\tau_{\infty}\right)=0$; this corresponds to physical time $t=\infty$. The condition $n_{1}\left(\tau_{\infty}\right)=0$ leads to three regimes of behavior for the clueless fraction $c_{\infty}$ (Fig. 2(a)). For $\lambda<1$ (subcritical), adopters abandon the fad sufficiently slowly that the fad can spread globally before dying out. In the supercritical regime of $\lambda>1$, adopters abandon the fad so quickly that there little time for the innovation to spread before it is extinguished. In this limit, Eq. (10) gives $\tau_{\infty}=\rho /(\lambda-1)$ and $c_{\infty}=1-\rho /(\lambda-1)$ to leading order, while for $\lambda=\lambda_{c}=1$, $c_{\infty}=1-\sqrt{2 \rho}$. Thus $c_{\infty}$ undergoes a continuous transition (in the $\rho N \gg 1$ limit) as $\lambda$ passes through the critical value $\lambda_{c}=1$ (Fig. 3(a)).

Let us now investigate the role of reinforcement on the dynamics of a fad. We consider the simplest situation of a single intermediate state; that is, $M=2$, or equivalently, three internal states for each individual. In this case, the evolution of $n_{0}$ and $n_{1}$ are again given by Eq. (4), while the solution of $n_{2}$ is

$$
n_{2}=1-(1-\rho)(1+\tau) e^{-\tau}-\lambda \tau .
$$

A curious feature of this result is that the density of fad adopters $n_{2}(\tau)$ can first decrease, then increase later on, before ultimately vanishing (Fig. 2(b)). This unusual behavior stems from the delicate interplay between abandonment of the fad and the creation of new adopters from the remaining reservoir of susceptible individuals. As a result of the 

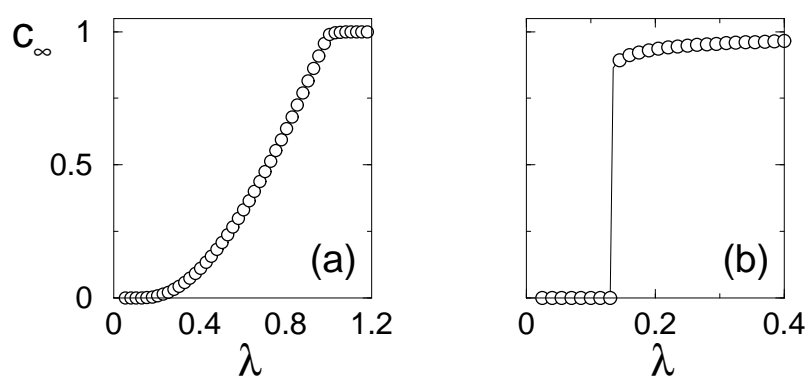

Figure 3. Clueless fraction $c_{\infty}$ versus abandonment rate $\lambda$ for: (a) two-state and (b) three-state models. The initial adopter fraction is $n_{1}(0)=10^{-4}$ in (a) and $n_{2}(0)=10^{-2}$ in (b).

two extrema in $n_{2}$ as a function of $\tau$, the stopping condition $n_{2}\left(\tau_{\infty}\right)=0$ can have one, two, or three roots, depending on $\lambda$. This change in the number of roots ultimately causes the discontinuity in the clueless fraction $c_{\infty}$ as a function of $\lambda$.

To locate this transition in the supercritical case equation, notice that (11) has three roots. We are interested in the smallest root and therefore expand the left-hand side of Eq. (11) for small $\tau$. Keeping the leading terms gives

$$
n_{2}\left(\tau_{\infty}\right) \approx \rho+\frac{1}{2} \tau_{\infty}^{2}-\lambda \tau_{\infty}=0 .
$$

From this quadratic equation, we see that the interesting behavior arises when $\lambda=\mu \sqrt{\rho}$ where $\mu=\mathcal{O}(1)$. With this convenient parameterization, the solution for $\tau_{\infty}$ is $\tau_{\infty}=\sqrt{\rho}\left[\mu \pm \sqrt{\mu^{2}-2}\right]$. Using the physically relevant smaller solution, we find, for $\mu>\mu_{c}$ (which equals $\sqrt{2}$ to lowest order)

$$
c_{\infty}=(1-\rho) e^{-\tau_{\infty}} \simeq 1-\sqrt{\rho}\left(\mu-\sqrt{\mu^{2}-2}\right) ;
$$

i.e., the clueless fraction is close to one (Fig. 3(b)). In the subcritical case, $\mu<\sqrt{2}$, the relevant root of $n_{2}\left(\tau_{\infty}\right)=0$ is $\tau_{\infty}=1 /(\mu \sqrt{\rho})$ to leading order. The clueless fraction is

$$
c_{\infty}=e^{-\tau_{\infty}}=e^{-1 /(\mu \sqrt{\rho})},
$$

which is close to zero for $\rho \rightarrow 0$. Thus the clueless fraction undergoes a first-order transition as a function of $\lambda$.

\section{Fad Completion Time}

A striking aspect of our fad model is that the time for a fad to die out has a nonmonotonic dependence on the abandonment rate $\lambda$ (Fig. 4). This non-monotonicity has a simple qualitative origin. If the abandonment rate is large, then the initial adopters abandon before they can recruit new adopters. Thus the fad quickly disappears. Conversely, if the abandonment rate is small, essentially the entire population adopts the innovation en masse, after which the fad disappears in a time that scales as $1 / \lambda$. Between these two limits, the fad "smolders" rather than just extinguishing itself immediately or suddenly igniting and then quickly extinguishing itself. In this intermediate range of 
$\lambda$ values, new adopters are slowly replenished at nearly the same rate as other adopters abandon the fad, so that the fad can be extremely long lived.

In a population of size $N$, we determine the time for a fad to end not by the criterion $\tau=\tau_{\infty}$, where the number of adopters vanishes, but rather by $n_{M}\left(\tau^{*}\right)=\frac{1}{N}$. Namely, only a single adopter remains in a finite population. This internal time corresponds to the value $T=\int_{0}^{\tau^{*}} d \tau / n_{M}(\tau)$ for the physical time at which the fad disappears. The actual determination of the completion time is very different for the cases $M=1$ and $M>1$, and we investigate these two cases in turn.
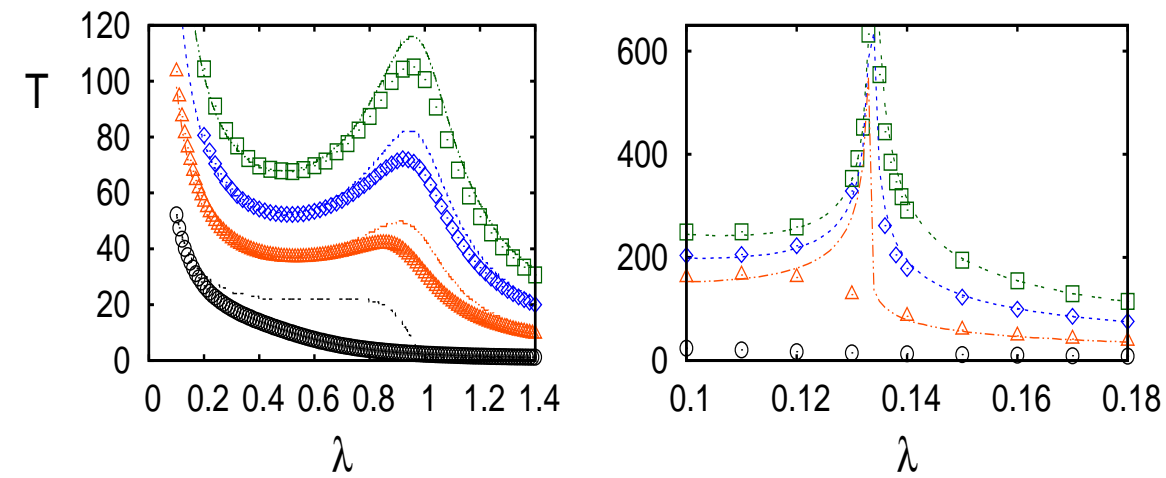

Figure 4. Completion time $T$ versus $\lambda$ for the fad model, for $M=1$ (left) and $M=2$ (right) with $\rho=10^{-2}$. Points are simulation results for $N=10^{2}, 10^{4}, 10^{6}, 10^{8}$ (bottom to top). Dashed lines are the corresponding results from numerically integrating the rate equations. For $M=1$, the width of the peak at $\lambda_{c}=1$ scales as $\sqrt{\rho}$.

\subsection{No reinforcement, $M=1$}

Generically, $T$ is proportional to $\ln N$ because $n_{1}$ goes to zero with a finite slope as $\tau$ approaches $\tau_{\infty}$ (Fig. 2(a)). As a consequence, the integral for $T$ is logarithmically divergent in $N$. However, the details of this dependence depends on the value of the abandonment rate $\lambda$.

In the subcritical regime $(\lambda<1)$, we determine $T$ by expanding $n_{1}$ about $\tau_{\infty}$ and using the condition $e^{-\tau_{\infty}}+\lambda \tau_{\infty}=1$ to obtain

$$
T=\frac{1}{\lambda+\lambda \tau_{\infty}-1} \int_{1 / N} \frac{d y}{y}=\frac{\ln N}{\lambda+\lambda \tau_{\infty}-1} .
$$

The lower limit in Eq. (15) follows from the stopping criterion $n_{1}\left(\tau^{*}\right)=\frac{1}{N}$, while the upper limit is immaterial for the asymptotic behavior.

In the supercritical regime $(\lambda>1)$, the density of adopters $n_{1}$ decreases almost linearly in $\tau$ over the entire range for which $n_{1}$ is positive. In this case [22], an expansion of $n_{1}$ about $\tau_{\infty}$ leads to $T=\ln (\rho N) /(\lambda-1)$.

In the critical case of $\lambda=1, n_{1}$ decreases quadratically with $\tau$ and the same expansion procedure as outlined above gives $T=\ln (\rho N) / \sqrt{2 \rho}$ in the asymptotic limit. Consistent with the logarithmic dependence at the critical point for the case $\rho=1 / N$, 
we find completion time distribution has a power law tail, $P(T) \sim T^{-2}$. The resulting average completion time is $T=\frac{1}{3} \log N$, a behavior that was obtained previously in the context of epidemic dynamics [23].

\subsection{Reinforcement, $M>1$}

In this case, the fad evolution in the supercritical regime closely mirrors the behavior of the $M=1$ case. In particular, the time dependence of $n_{M}(\tau)$ is similar to $n_{1}(\tau)$ in the case of no reinforcement: $n_{M}$ approaches zero with finite slope, from which the ending time of the fad again scales as $\ln N$. However, in constant to the case $M=1$, the value of $\tau$ where $n_{M}(\tau)$ first reaches zero changes discontinuously as $\lambda$ passes through $\lambda_{c}$ (Fig. 2(b)). More interestingly, when $\lambda \approx \lambda_{c}, n_{M}$ approaches zero with a quadratic minimum, as illustrated in the inset to Fig. 2(b). This property leads to an algebraic, rather than a logarithmic, dependence of the completion time on $N$. Finally for $\lambda<\lambda_{c}$, $n_{M}$ again reaches zero with a finite slope, leading to a logarithmic dependence of the ending time on $N$. Thus the time for the fad to disappear at the critical point is much larger than the corresponding ending times away from this point. Monte Carlo simulations of the fad dynamics in a finite population mirror our analytic predictions, except near the first-order transition, where large fluctuations arise.

Let us now focus on the properties of the completion time at the first-order transition point where fluctuations are particularly strong. There are two independent and natural scenarios for which to define the lifetime of the fad: (i) a fixed number of initial adopters (generally we treat the case of one adopter) or (ii) a fixed fraction $\rho$ of initial adopters. To find the fad lifetime in the former case of $\rho=\frac{1}{N}$, it is again convenient to use parameterization $\lambda=\mu \sqrt{\rho}$ because the critical value of $\mu$ is $\mathcal{O}(1)$. We therefore substitute the critical value $\mu_{c}=\sqrt{2}$ (to lowest order) into the expansion (12) for $n_{2}$ to obtain $n_{2}=\frac{1}{2}(\sqrt{2 \rho}-\tau)^{2}$. The ending time for the fad is now given by

$$
T=2 \int_{0}^{\tau^{*}} \frac{d \tau}{(\sqrt{2 \rho}-\tau)^{2}},
$$

with $\tau *$ determined from the criterion $n_{2}\left(\tau^{*}\right)=\frac{1}{N}$. The latter gives $\tau^{*}=\sqrt{2 \rho}-\sqrt{2 / N}$ and using this upper limit in (16) gives $T=\sqrt{2 N}$. However, the prefactor arises from the imprecise criterion $n_{2}\left(\tau^{*}\right)=\frac{1}{N}$, and simulations instead give $T \sim 0.56 \sqrt{N}$. For a fixed fraction of initial adopters $\rho$, our simulations show that the average fad lifetime grows with $N$ roughly as $N^{1 / 4}$ for $M=2$, a result for which we do not yet have an explanation.

As a result of the large fluctuations near the transition, the completion time distribution consists of distinct components. One component corresponds to realizations where the fad quickly dies out so that the population is almost entirely clueless (Fig. [5). In contrast, for the remaining fraction of realizations, nearly everyone adopts and then abandons the fad. Corresponding to this dichotomy in the fate of individual realizations, the distribution of times at which the fad disappears has distinct short-lived and longlived contributions (Fig. 6) . 

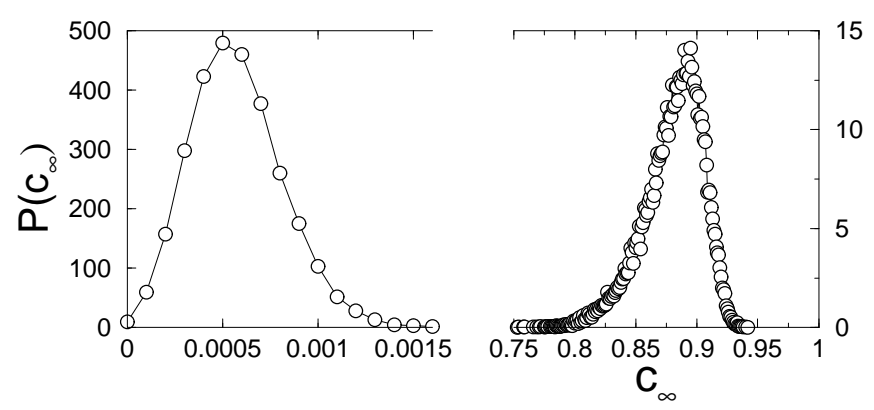

Figure 5. Probability density $P\left(c_{\infty}\right)$ for the fraction of clueless individuals at the end of the process at the critical point, $\lambda_{c}=\sqrt{2 \rho}$, for the $M=2$ fad model, with initial density $\rho=10^{-2}$ and population size $N=10^{4}$. (Note the horizontal scale break.)
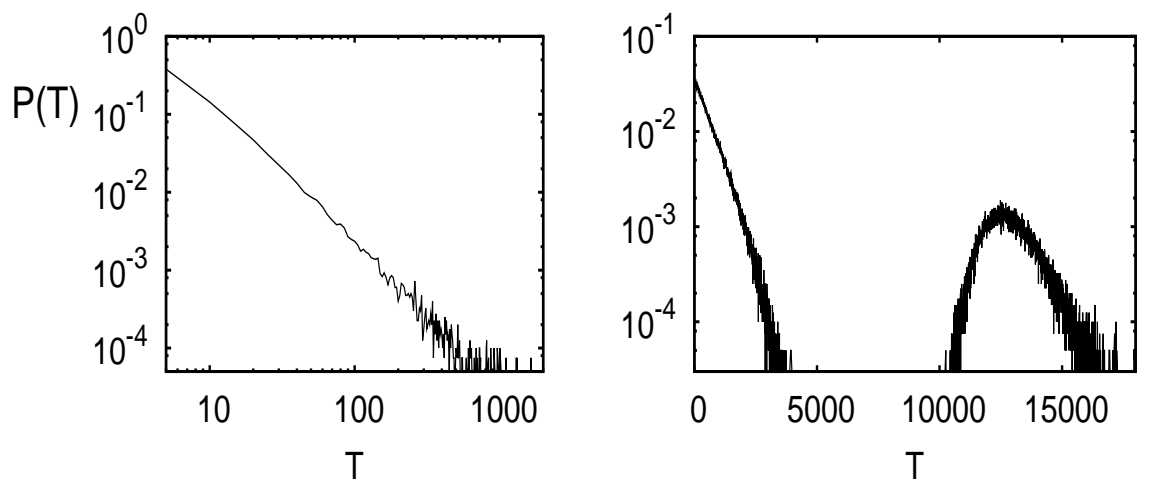

Figure 6. Probability distribution of completion time $T$ for the fad model at the critical point, for $M=1$ (left) and $M=2$ (right) with $\rho=1 / N$. We use $N=10^{9}$ for $M=1$ and $N=10^{6}$ for $M=2$.

\section{Summary}

We have shown how the mechanism of social reinforcement plays a strong role in determining how permanent innovations and transient fads are adopted in a socially-interacting population. For permanent innovations, we modeled the effect of reinforcement by endowing each individual with $M+1$ levels of awareness $0,1,2, \ldots, M$. An individual increases his/her level of awareness by one unit as a result of interacting with an adopter, and adoption occurs when an individual reaches the highest awareness level $M$. In the mean-field limit, we found that the time for the innovation to be adopted universally scales as $N^{1-1 / M}$, so that increasing $M$ delays the onset of the innovation.

For transient fads, the fad quickly disappears for $\lambda>\lambda_{c}$, while for $\lambda<\lambda_{c}$ the fad is nearly universally adopted before finally being forgotten. The fad lasts the longest when $\lambda=\lambda_{c}$. Here new adopters are slowly replenished as others abandon, so that the fad slowly smolders rather than igniting and quickly burning out. The transition in the fraction of clueless individuals - those who have no knowledge of the fad before it disappears - is second order when there is no reinforcement, but first order with 
reinforcement. The rich phenomenology near the transition may offer opportunities to help predict the reach of a technological innovation before it is released on the market. A fruitful direction for additional research is to include the effect of stochastic fluctuations and heterogeneous social connections. Both of these attributes can be anticipated to considerably enrich the dynamics that have been uncovered in this work.

\section{Acknowledgments}

We thank Damon Centola for helpful discussions, Sam Bowles for literature advice, and NSF grants CCF-0829541 (PLK) and DMR-0906504 (DV and SR) for financial support.

\section{References}

[1] N. T. J. Bailey, The Mathematical Theory of Infectious Diseases and Its Applications (Oxford University Press, Oxford, 1975); R. M. Anderson and R. M. May, Infectious Diseases of Humans: Dynamics and Control (Oxford University Press, Oxford, 1992).

[2] J. Coleman, E. Katz, and H. Menzel, Sociometry 20, 253 (1957).

[3] F. M. Bass, Mgmt. Sci. 15, 215 (1969).

[4] F. M. Bass, J. Business 53, S51 (1980).

[5] S. Morris, Rev. Econ. Studies 67, 57 (2000).

[6] E. M. Rogers, Diffusion of Innovations (Free Press, New York, 2003).

[7] M. Granovetter, Am. J. Sociol. 83, 1420 (1978).

[8] S. Lohmann, World Politics 47, 42 (1994).

[9] D. Centola, Science 329, 1194 (2010). For theoretical background see D. Centola, R. Wilker, and M. W. Macy, Am. J. Sociol. 110, 1009 (2005); D. Centola, V. M Eguiluz, and M. W. Macy, Physica A 374, 449 (2007).

[10] S. N. Majumdar and P. L. Krapivsky, Phys. Rev. E 63, 045101(R) (2001).

[11] P. S. Dodds and D. J. Watts, Phys. Rev. Lett. 92, 218701 (2004).

[12] H. P. Young, Amer. Econ. Rev. 99, 1899 (2009).

[13] C. de Kerchove, G. Krings, R. Lambiotte, P. Van Dooren, and V. D. Blondel, Phys. Rev. E 79, 016114 (2009).

[14] B. Karrer and M. E. J. Newman, Phys. Rev. E 82, 016101 (2010).

[15] J.-P. Onnela and F. Reed-Tsochas, Proc. Natl. Acad. Sci. USA 107, 18375 (2010).

[16] F. J. Pérez-Reche, J. J. Ludlam, S. N. Taraskin, and C. A. Gilligan, Phys. Rev. Lett. 106, 218701 (2011).

[17] If one uses the criterion that the innovation is complete when $n_{1}=1$, one obtains the unphysical result that the completion time is infinite.

[18] J. Kaldasch, Physica A 390, 2692 (2011).

[19] Details will be given in P. L. Krapivsky, S. Redner, and D. Volovik, in preparation.

[20] The clueless are analogous to the population susceptible fraction in rumor-spreading models, see D. P. Maki and M. Thompson, Mathematical Models and Applications (Prentice-Hall, Englewood Cliffs, NJ, 1973); B. Hayes, American Scientist 93, 207 (2005).

[21] P. L. Krapivsky, S. Redner, and E. Ben-Naim, A Kinetic View of Statistical Physics (Cambridge University Press, Cambridge, England 2010).

[22] Here we assume $\rho \ll 1$ and $\rho N \gg 1$. In the extreme case of $\rho=N^{-1}$, fluctuations are artificially large and the rate equation approach becomes invalid.

[23] E. Ben-Naim, P.L. Krapivsky, Phys. Rev. E 69, 050901R (2004). 